\title{
55. Information Methods for Predicting Risk and Outcome of Stroke
}

\author{
Linda Liang, Rita Krishnamurthi, Nikola Kasabov, Valery Feigin
}

Stroke is a major cause of disability and mortality in most economically developed countries. It is the second $\mathrm{CE}^{0}$ leading cause of death worldwide (after cancer and heart disease) $[55.1,2]$ and a major cause of disability in adults in developed countries [55.3]. Personalized modeling is an emerging effective computational approach, which has been applied to various disciplines, such as in personalized drug design, ecology, business, and crime prevention; it has recently become more prominent in biomedical applications. Biomedical data on stroke risk factors and prognostic data are available in a large volume, but the data are complex and often difficult to apply to a specific person. Individualizing stroke risk prediction and prognosis will allow patients to focus on risk factors
55.1 Introduction .............................. 1

55.2 Literature Review .......................... 2

55.2 .1 Stroke............................. 2

55.2.2 Information Methods for Predicting Risk and Outcome of Stroke

55.3 Conclusion

References ......................................... 7

specific to them, thereby reducing their stroke risk and managing stroke outcomes more effectively. This chapter reviews various methods-conventional statistical methods and computational intelligent modeling methods for predicting risk and outcome of stroke.

\subsection{Introduction}

The $\mathrm{TS}^{1} \mathrm{TS}^{2}$ human brain is a control center, an intricate master computer that controls and integrates various organ systems of the body. The nerve cells of the brain are connected with other cells in every part of the body. They deliver messages from the brain to tell the organ systems and the brain itself how to function, communicate, form memories, or make decisions. They also send messages back to the brain, telling it what is happening throughout the body. The brain is a complex system which evolves its functions and structures during its lifetime [55.4]. In the brain, there are complex interactions between genes and neuronal functions.

However, abnormalities in some of these interactions might cause brain diseases, such as brain cancer, Parkinson's disease, and Alzheimer's disease, etc. Stroke is a prevalent brain disease and has become a major public health challenge and concern in New Zealand, as well as globally. As proposed by Tobias et al. [55.5], in New Zealand, over 7000 people each

year will experience a stroke event, and at least threequarters of this population will die or be dependent on others for health care 1 year after stroke.

Until now, many intelligent systems have been developed with the purpose of improving health care and providing better health care facilities at a reduced cost. However, a review of the literature on stroke occurrence and outcome shows that traditional predictive models using standard population statistics can only apply to a group of people and are unable to predict the degree of risk occurrence or disability level for either an individual person at risk of stroke or a stroke survivor. These conventional statistical methods of prediction employ only the most significant predictive variables so that less statistically significant personal information that may be clinically significant for a particular person but not for a group of people is certainly lost [55.6]. For that reason, the concept of personalized modeling may indeed be an ideal approach 
Part K | Information Modelling for understanding and curing Brain Diseases

worth exploring and integrating into the medical system for diagnosis, prediction, and management. Personalized modeling is an emerging effective approach for knowledge discovery in biomedical applications. The principle of this computational intelligent approach is to create a personalized diagnostic or prediction model for an individual person based on his/her nearest neigh-

\subsection{Literature Review}

\subsubsection{Stroke}

What Is a Stroke?

The World Health Organization (WHO) defines stroke as [55.7]:

rapidly developing clinical signs of focal or global disturbance of cerebral function lasting more than 24 hours (unless interrupted by surgery or death) with no apparent cause other than of vascular origin.

It is generally accepted that the lifetime risk of stroke occurrence is 1 in 6 , at least as high as the risk for developing Alzheimer's disease [55.8].

Stroke has also a large physical, psychological and financial impact on patients/families, the health care system, and society $[55.9,10]$. Lifetime costs per stroke patient range from US $\$ 59.8 \mathrm{~K}$ to US $\$ 230 \mathrm{~K}$ [55.10]. The majority (about $75 \%$ ) of cases of stroke occur in people over the age of 65 years [55.11, 12], and about one third of patients die of stroke within a year of onset $[55.13,14]$. Over half of the survivors remain dependent on others for everyday activities, often with significant adverse effects on caregivers [55.15].

Their family members are also affected due to the suffering of their loved ones as well as by the burden of caring for them, uncertain about future plans and anxious about increased financial burdens for the patient's treatment.

What Are the Risk Factors?

There are about 200 factors that can increase the risk of a stroke but the most important ones are:

- Elevated blood pressure

- Smoking

- Diabetes mellitus

- Increasing age

- Overweight (especially abdominal obesity)

- High blood cholesterol level bors of predictive variables that are pertinent to that person.

The objectives of this chapter are to provide a brief introduction to stroke and then review various information methods, including conventional statistical methods and computational intelligent modeling methods for predicting risk and outcome of stroke.
- Poor, unbalanced diet lacking fruit and vegetables

- Sedentary lifestyle

- Heart rhythm problems such as atrial fibrillation

- History of heart disease.

What Are the Symptoms?

The signs and symptoms of a stroke differ and depend on the area of the brain affected and the amount of brain tissue damaged. Small strokes may not cause any significant focal neurological symptoms (so-called silent strokes). However, when accumulated, they may lead to clinically significant consequences, such as vascular dementia. In general, stroke in the left side of the brain has clinical symptoms on the right side of the body, whereas the left side of the body is affected by a stroke in the right side of the brain.

According to the US National Institute of Neurological Disorders and Stroke (NINDS), the common symptoms of stroke generally come suddenly and may include:

- Sudden loss of consciousness: the victim may become stuporous or hard to arouse.

- Sudden loss of vision: difficulty with seeing in one or both eyes, such as blurred vision.

- Sudden headache: sudden onset severe headache that may be accompanied by vomiting or dizziness (loss of balance)

- Sudden trouble with muscle movements: difficulty with walking, moving the arm or leg on one side of body, carrying or picking up objects.

- Sudden trouble with speaking and understanding: may have problems with thinking or forming speech, such as when speaking, the words sound fine but do not make sense.

How Does Stroke Happen?

Stroke is a heterogeneous disorder that consists of two major pathological types (ischemic and hemorrhagic), and each type has different subtypes with different 


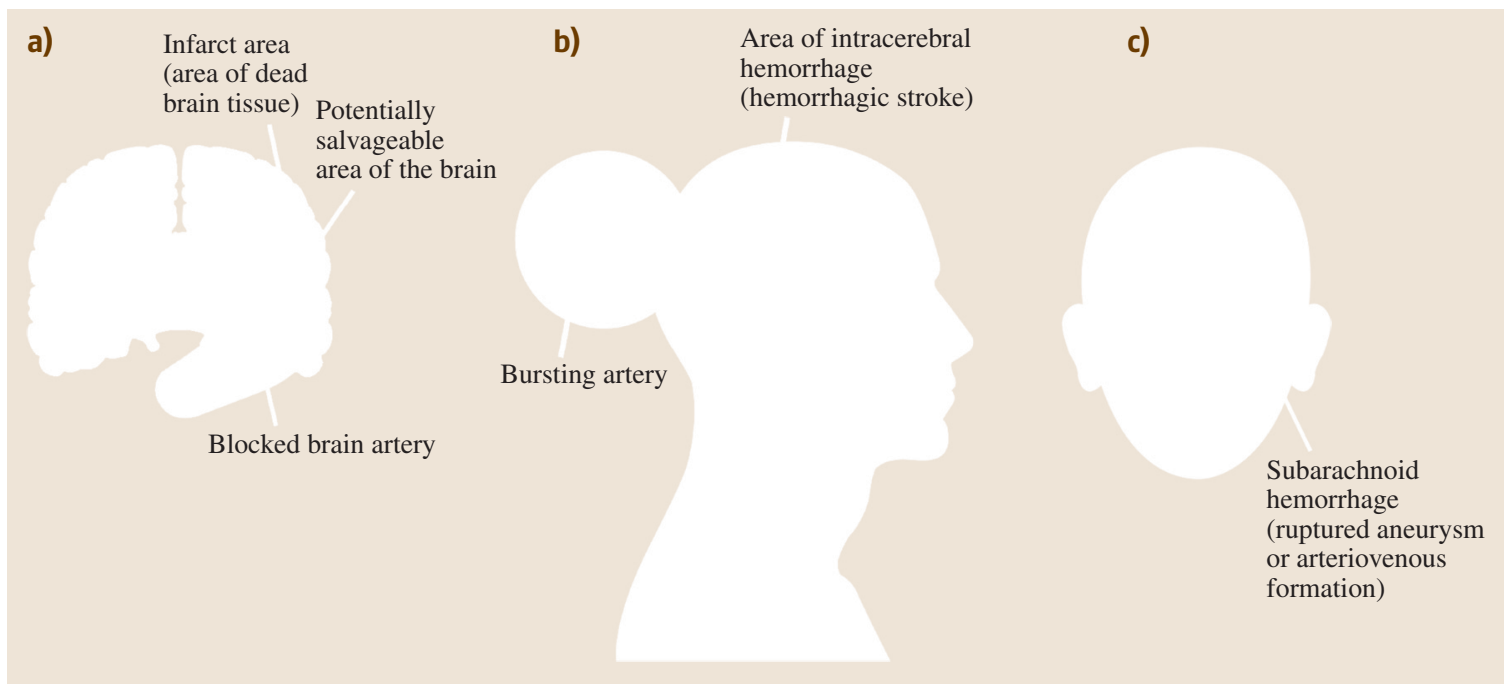

Fig. 55.1 (a) Ischemic stroke occurs when a blood vessel in the brain becomes blocked [55.16]. (b) Intracerebral hemorrhage occurs when blood vessels within the brain become damaged and burst within the brain [55.16]. (c) Subarachnoid hemorrhage occurs when a cerebral aneurysm ruptures; blood will fill the space surrounding the brain [55.16]

causes and outcomes. The two major types of stroke are described below (Fig. 55.1a-c).

Ischemic Stroke. Ischemic stroke is the most common type of stroke, accounting for almost $85 \%$ of all stroke cases. It occurs when there is a blood clot in the blood vessel of the brain that reduces or blocks the blood supply coming from the heart to the brain. As there is no nutrient/energy storage in the brain, it needs a constant supply of nutrients from the blood. The blood carries sugar and oxygen to the brain and takes away cellular waste and carbon dioxide. If an artery is blocked, the brain cells cannot receive the required level of oxygen and glucose needed, thus the affected cells begin to shut down. If there is no blood supply for as little as $7 \mathrm{~s}$, the affected brain cells may die.

Based on different mechanisms of stroke, ischemic stroke consists of at least 4 subtypes: cardioembolic stroke, ischemic stroke due to large artery disease (such as atherosclerosis), ischemic stroke due to small artery disease (such as hypertension, intracranial arteritis), and ischemic stroke due to hematological disorders and other rare conditions.

Hemorrhagic Stroke. Hemorrhagic stroke accounts for up to $15 \%$ of all stroke cases. It often happens when an artery bursts and bleeds into the brain (intracerebral hemorrhage) or around the brain (subarachnoid hemorrhage).
Intracerebral Hemorrhage. Intracerebral hemorrhage is a type of stroke caused by the breaking of a diseased blood vessel in the brain, leading the blood to leak into the brain tissue. This may lead to direct (destroying brain cells) or indirect damage of the affected brain cells due to a sudden build-up in the intracranial pressure, each of which may lead to unconsciousness, lost neurological function, or even death. Intracerebral hemorrhage may be caused by different mechanisms (e.g., elevated blood pressure, amyloid angiopathy) in different parts of the brain (e.g., supratential, infratentirial $\mathrm{CE}^{3}$ hemorrhage), each of which carries a different prognosis and requires different management strategies.

Subarachnoid Hemorrhage. Subarachnoid hemorrhage is the result of a blood vessel bursting in the area between the brain and the thin tissues that surround the brain. This area is called the subarachnoid space, which is the area outside of the brain tissue. Typical symptoms of a patient with subarachnoid hemorrhage include loss of consciousness, vomiting, severe headache or neck pain, and neck stiffness. Subarachnoid hemorrhage most often results from a rupture of an intracranial aneurysm but it may also be caused by a rupture of the brain artery due to other causes (so-called nonaneurysmal subarachnoid hemorrhage). The management of aneurysmal subarachnoid hemorrhage is very much different from the management of nonaneurysmal subarachnoid hemorrhage. 
$4 \quad$ Part K $\mid$ Information Modelling for understanding and curing Brain Diseases

\subsubsection{Information Methods for Predicting Risk and Outcome of Stroke}

To date, a number of technologies have been adopted to predict stroke occurrence and outcomes. These technologies can be divided into two major categories: conventional statistical methods and computational intelligent machine learning methods.

\section{Conventional Statistical Methods}

Currently conventional statistical methods are more widely used and commonly applied for stroke prediction data analysis. For example, descriptive statistics (e.g., frequency statistics) has been used to calculate the frequency of strokes in the general population, across gender, and ethnic groups etc. [55.17]; the correlation method (e.g., Spearman rank correlation) was utilized to compute the correlation between two different scales, such as the Barthel index and SF-36 [55.18]; logistic regression was applied to analyze the factors associated with the SF-36 subscales in order to discover which of these variables best discriminate between patients with low and high scores on the SF-36 subscales [55.19]; and one-way of variance and $\chi^{2}$ (chi square) test have been adopted to assess the differences between different stroke outcomes [55.20].

However, conventional statistical methods have limitations in efficiency and prediction accuracy compared to machine learning methods. Khosla and his colleagues [55.21] present an integrated machine learning approach to compare the Cox proportional hazards model (one of the most commonly used conventional statistical methods in medical research) on the Cardiovascular Health Study (CHS) dataset for stroke risk prediction. Their research demonstrated that machine learning methods significantly outperform the Cox model in terms of stroke risk estimation.

\section{Machine Learning Methods}

In [55.22]:

Machine learning is the process of discovering and interpreting meaningful information, such as new correlations, patterns and trends by sifting through large amounts of data stored in repositories, using pattern recognition technologies as well as statistical and mathematical technique.

In other worlds, machine learning is a process of using different analysis techniques to observe previously unknown, potentially meaningful information, and discover strong patterns and relationships from a large dataset that can be applied most accurately to a particular person. Kasabov [55.23] classified computational models into three categories (e.g., global, local, and personalized), which have being widely used in the areas of data analysis and decision support in general, and in the areas of medicine and bioinformatics in particular. A review study shows that the personalized modeling approach generally outperforms the conventional statistical methods for prediction or classification of conditions.

Personalized Modeling. The concept of personalized modeling is one type of local modeling that is created for every single new input vector of the problem space based on its nearest neighbors using the transductive reasoning approach [55.23]. The basic philosophy behind this approach when applied to medicine is that every person is different from others, thus he/she needs and deserves a personalized model and treatment that best predicts possible outcomes for this person. This way of reasoning is much closer to a way of clinical decision making that is used by clinicians in their everyday practice as opposed to a group-based approach utilized by conventional statistical methods.

Personalized modeling is a novel and effective method that has been applied for evaluating and dealing with a variety of modeling problems. For instance, in the field of personalized health care, the knowledge uncovered by this approach has significantly contributed to prediction, diagnosis, and therapy for individual patients' diseases. In the articles by Ginsburg and $\mathrm{Mc}$ Carthy [55.24] and TEMU [55.25], it is mentioned that providing a personalized therapy for an individual patient during the diagnosis time frame has proved to be very efficient and helpful.

Nowadays, the concept of personalized medicine is becoming a leading trend in medicine, health care, and life science. As presented by Lesko [55.26], from US Food and Drug Administration

Personalized medicine can be viewed... as a comprehensive, prospective approach to preventing, diagnosing, treating, and monitoring disease in ways that achieve optimal individual healthcare decisions.

As stated by the Personalized Medicine Coalition [55.27], traditional medicine is primarily based on the visible symptoms of the disease, but recently doctors have been able to integrate an individual patient's molecular profile to characterize various forms of can- 
cer (e.g., breast cancer, brain cancer, and liver cancer, etc.) to make a decision about treatment. Furthermore, according to Ginsburg and McCarthy [55.24], the objective of personalized medicine is to determine a patient's disease at the molecular level, so the right therapies can be applied to the right people at the right time. The personalized medicine approach is being increasingly used to accommodate the individual patient's molecular/genetic profile.

Currently, there are several methods of personalized modeling, which are outlined below.

K Nearest Neighbor (KNN). The simplest method of personalized modeling was originally proposed by Fix and Hodges in 1951 [55.28]. KNN is a supervised learning algorithm that has been successfully used for classifying sets of samples based on nearest training samples in a multi-dimensional feature space. lows:

The basic idea behind the KNN algorithm is as fol-

- Firstly, a set of pair features (e.g., $\left(x_{1}, y_{1}\right), \ldots$, $\left.\left(x_{n}, y_{n}\right)\right)$ are defined to specify each data point, and each of those data points are identified by the class labels $C=\left\{c_{1}, \ldots, c_{n}\right\}$.

- Secondly, a distance measure is chosen (e.g., Euclidean distance or Manhattan distance) to measure the similarity of those data points based on all their features.

- Finally, the $k$-nearest neighbors are found for a target data point by analyzing similarity and using the majority voting rule to determine which class the target data point belongs to.

Weighted Nearest Neighbor (WKNN). This is designed based on the transductive reasoning approach, which has been widely used to evaluate the output of a model focusing on solely an individual point of a problem space using information related to this point [55.29]. In the WKNN algorithm, each single vector requires a local model that is able to best fit each new input vector rather than a global model, thus each new input vector can be matched to an individual model without taking any specific information about existing vectors into account.

In contrast to the KNN algorithm, the basic idea behind the WKNN algorithm is that the output of a new input vector is calculated not only depending upon its k-nearest neighbor vectors, but also upon the distance between the existing vectors and the new input vector, which is represented as a weight vector $w$.
Weighted Distance and Weighted Variables K Nearest Neighbor (WWKNN). This is a novel personalized modeling algorithm, which was proposed by Kasabov in 2007. The basic idea behind this algorithm is as follows: the output of each new input vector is measured not only depending upon its $k$-nearest neighbors, but also upon the distance between the existing vectors and the new input vectors, and also the power of each vector which is weighted according to its importance within the subspace (local space) to which the new input vector belongs. We start with the assumption that all the variables from a data set are used and the distance of vectors is calculated in a $\mathrm{V}$-dimensional space with all input variables having the same impact on the output variables. However, the different variables may vary in importance when classifying vectors if these variables are ranked by their discriminative power in classifying vectors over the entire V-dimensional Euclidean space. As a result, it can be seen that variables may have a different ranking when we measure the discriminative power of the same variables for a subspace of the problem space. The output of each new input vector can be calculated by using this type of ranking within the neighborhood of $k$-nearest neighbor vectors.

Evolving Spiking Neural Networks (eSNN). The brain is the center of the nervous system, it is an extremely complex organ. The cerebral cortex of the human brain contains roughly $15-33$ billion neurons, depending on gender and age, linked with up to 10000 synaptic connections each.

A typical neuron in the human brain is sketched in Fig. 55.2. A typical neuron can be divided into three functionally distinct parts, called dendrites, soma, and axon. Generally speaking, the dendrites play the role of the input devices that collect signals from other neurons and transmits them to the soma. Soma is the main part of the neuron containing the genetic information: if the total input exceeds a certain threshold, then an output signal is generated. The output signal is taken over by the output devices, the axons, which deliver the signal to other neurons or other parts of the body. The junction between two neurons is called a synapse. It is used to transfer signals between two neurons.

The remarkable information processing capabilities of the brain have inspired numerous mathematical abstractions of biological neurons. A neural network (NN) known as an artificial neural network (ANN) is defined as a hardware or software computational model that is inspired by the biological nervous systems, such as the brain, and processes $\mathrm{CE}^{4}$ information. Many ANN 


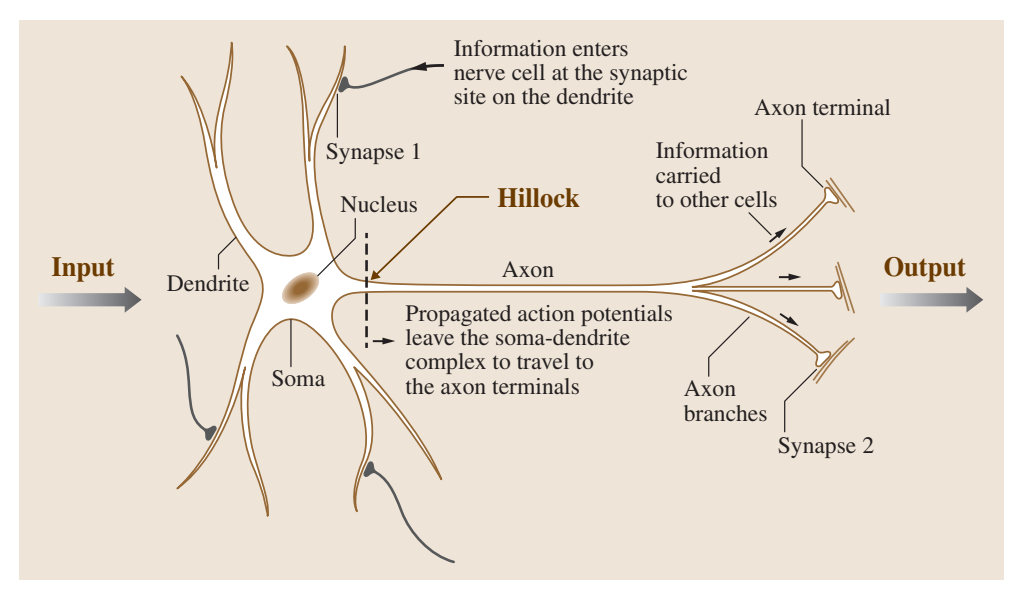

Fig. 55.2 Schematic drawing of a typical neuron [55.31]

models have been successfully developed and applied across many disciplines, including classification, time series prediction, pattern recognition, and so on [55.30]. However, current ANN models do not provide good performance when applied to complex stochastic and dynamic processes such as modeling brain diseases. For this reason, new ANN models should be developed in order to become more accurate and efficient in knowledge discovery and information processing.

Maass [55.32] classifies past and current neural network models into three generations. Spiking neural networks (SNN) is the third generation of neural network models, which is a complex and biologically plausible connectionist model. In biological systems neurons are connected at synapses, and signals transfer information from one neuron to another. Quite a few models of SNN have been developed so far: Hodgkin-Huxley's model [55.33]; Spike response models [55.34-36]; integrate-and-fire models [55.35, 37]; Izhikevich models [55.38-40], etc.

SNN has been increasingly applied in the field of science and engineering as in other disciplines to solve complicated prediction and classification problems, such as speech recognition [55.41], audio and video analysis [55.42, 43], and financial forecasting [55.44]. In recent years, SNN has become a powerful computational tool that has been widely adopted for diagnosing and monitoring the prognosis of a disease, as evidenced by over 500 published papers each year featuring neural network applications in medicine [55.45]. In medical practice, SNN has been successfully used for diagnosing breast cancer [55.46], making prognosis for patients with congestive heart failure [55.47], and predicting the risk of death for lung cancer patients [55.48].
More recently, spiking neural networks have been successfully used for the prediction of functional outcome associated with clinical variables that are associated with rehabilitation in stroke [55.6]; early diagnosis of ischemic stroke [55.49] and classifying the gait patterns of post-stroke patients into homogenous groups [55.50].

Evolving SNN (eSNN), which is an SNN approach, was first proposed by Wysoski et al. in 2006 [55.51]. It consists a set of SNN that evolve their structure through incremental and fast one-pass learning from data.

Space and time can be viewed as the important aspects of all real-world phenomena. Spatio-temporal data (STD) contains information relating space and time. According to Fayyad and Grinstein [55.52], nowadays, approximately $80 \%$ of the available datasets have spatial components and are often related to some temporal aspects. Examples of such data are environmental, audio/visual, medical, and brain signals. So far, most available stroke data are STD, thus a great challenge is how to process these complex STD.

In general, classical statistical and computational techniques are insufficient when they are applied to spatio-temporal datasets for the following reasons:

- Spatio-temporal datasets are embedded into continuous space, whereas the classical datasets are often discrete.

- The patterns in these datasets are often local, but classical techniques normally focus on global patterns.

- They model either space and time separately, or mix both components in a simple way, failing $\mathrm{CE}^{5}$ to capture essential relations between variables in the STD. 
eSNN is considered an emerging computational technique for the analysis of spatio-temporal datasets. As proposed by Kasabov [55.30], the development of a novel integrated evolving personalized modeling sys- tems by utilizing novel technology such as evolving spiking neural networks (eSNN) might facilitate more precise decision-making to ensure patients receive optimal prognosis and treatment.

\subsection{Conclusion}

The human brain is a complex network of interconnected cells (neural networks) that develops its functionalities and structures during the lifetime of a person. There are complex interactions between genes, the environment, and neuronal function within the brain. However, some of these interactions might become abnormal and cause brain diseases.

Stroke is a prevalent brain disease and has become a major public health concern in New Zealand, as in many other countries worldwide. A large volume of biomedical data on stroke risk factors and prognosis is available, but the interpretation of this data is complex and challenging. An increasing number of studies have attempted to explore these complex data, but most of these studies applied conventional statistical methods. Literature reviews show that the traditional predictive models using conventional statistical methods can only apply to a group of people and are unable to predict the degree of risk occurrence or disability level for either an individual person at risk of stroke or a stroke survivor. Therefore, there is a growing need for utilizing computational models to study this data, especially applied to estimating personalized risk to better understand the pathophysiology in individual and specific groups of stroke, and to achieve improved and reliable risk prediction for individuals.

Personalized modeling was recently proposed as a novel method for knowledge discovery in biomedical applications. The basic philosophy behind this computational intelligent approach when applied to medicine is that every person is different from others, thus he/she needs and deserves a personalized model and treatment that best predicts possible outcomes for this person. The principle of this approach is to create a personalized diagnostic or prediction model for an individual person based on his/her nearest neighbors of predictive variables that are pertinent for that person. For instance, employing eSNN might result in reducing the time, cost, medical error, and mortality rate. The concept of personalized modeling can be viewed as an ideal approach well worth exploring and integrating into the medical system for diagnosis, prediction, and prescription of various diseases, including stroke.

\section{References}

55.1 S.C. Johnston et al. TS $^{6}$ : Global variation in stroke burden and mortality: Estimates from monitoring, surveillance, and modelling, Lancet Neurol. 8, 345-354 (2009)

55.2 P.M. Rothwell: The high cost of not funding stroke research: A comparison with heart disease and cancer, Lancet Neurol. 357, 1612-1616 (2001)

55.3 M. Tobias et al. TS $^{6}$ : Modelling Stroke: A Multi-State Life Table Model, Occasional Bulletin/Public Health Intelligence (Ministry of Health, Wellington 2002)

55.4 N. Kasabov: Brain-, Gene-, and Quantum Inspired Computational Intelligence: Challenges and $0 p-$ portunities (Springer, Heidelberg 2007)

55.5 M. Tobias et al. Ts ${ }^{6}$ : Stroke surveillance: Population-based estimates and projections for New Zealand, Aust. N. Z. J. Public Health 31, 520525 (2007)

55.6 J. Wieslaw, et al. Ts ${ }^{6}$ : Neural network modeling accurately predicts the functional outcome of stroke survivors with moderate disabilities, Arch. Phys. Med. Rehabil. 78 340-345 (1997)

55.7 K. Aho, et al. Ts $^{6}$ : Cerebrovascular disease in the community: Results of a WHO collaborative study, Bull. World Health Organ. 58, 113-130 (1980)

55.8 S. Seshadri, A. Beiser, M. Kelly-Hayes, C.S. Kase, R. Au, W.B. Kannel, P.A. Wolf: The lifetime risk of stroke: Estimates from the Framingham Study, Stroke 37, 345-350 (2006)

55.9 K. Strong, et al. Ts $^{6}$ : Preventing stroke: Saving lives around the world, Lancet Neurol. 6, 182-187 (2007)

55.10 J.J. Caro, et al. Ts $^{6}$ : Management patterns and costs of acute ischemic stroke: An international study, Stroke 31, 582-590 (2000)

55.11 R. Bonita, et al. TS $^{6}$ : Stroke incidence and case fatality in Australasia. A comparison of the Auckland and Perth population-based stroke registers, Stroke 25, 552-557 (1994)

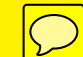


8 Part $\mathrm{K}$ | Information Modelling for understanding and curing Brain Diseases

55.12 R. Bonita, et al. TS 6 : Changes in stroke incidence and case-fatality in Auckland, New Zealand, 198191, Lancet 342, 1470-1473 (1993)

55.13 C.S. Anderson, et al. $\mathrm{Ts}^{6}$ : Predicting survival for 1 year among different subtypes of stroke. Results from the Perth Community Stroke Study, Stroke 25, 1935-1944 (1994)

55.14 R. Bonita, et al. TS $^{6}$ : Predicting survival after stroke: A three-year follow-up, Stroke 19, 669-673 (1988)

55.15 C.S. Anderson, et al. Ts $^{6}:$ A population-based assessment of the impact and burden of caregiving for long-term stroke survivors, Stroke 26, 843-849 (1995)

55.16 V. Feigin: When Lightning Strokes: An Illustrated Guide to Stroke Prevention and Recovery (HarperCollins, Auckland 2004)

55.17 V.L. Feigin, et al. $\mathrm{TS}^{6}$ : Ethnic disparities in incidence of stroke subtypes: Auckland regional community stroke study, 2002-2003, Lancet Neurol. 5, 130-139 (2006)

55.18 S.M. Lai, et al. TS $^{6}$ : Prediction of functional outcome after stroke: Comparison of the orpington prognostic scale and the NIH stroke scale, Stroke 29, 1838-1842 (1998)

55.19 M.L. Kauhanen: Quality of life after stroke: Clinical, functional, psychosocial and cognitive correlates, Neurology (University of Oulu, Oulu 1999)

55.20 V.L. Feigin, et al. Ts $^{6}$ : Auckland stroke outcomes study. Part 1: Gender, stroke types, ethnicity and functional outcomes 5 years poststroke., Neurology 75, 1597-1607 (2010)

55.21 A. Khosla, et al. Ts ${ }^{6}$ : An integrated machine learning approach to stroke prediction, 16th ACM SIGKDD Conf. Knowl. Discov. Data Min. (2010)

55.22 D.T. Larose: Discovering Knowledge in Data: An Introduction to Data Mining (Wiley, Hoboken 2005)

55.23 N. Kasabov: Evolving Connectionist Systems: The Knowledge Engineering Approach (Springer, London 2007)

55.24 G.S. Ginsburg, J.J. McCarthy: Personalized medicine: Revolutionizing drug discovery and patient care, Trends in Biotechnol. 19, 491-496 (2001)

55.25 TEMU: Personalised Medicine: Current Trends and Scientific Challenges (TEMU, TS $^{7} 2008$ ), online at: http://www.temu.gr/2008/sessions.html

55.26 L.J. Lesko: Personalized medicine: Elusive dream or imminent reality, Clin. Pharmacol. \& Ther. 81, 807-816 (2007)

55.27 Personalized Medicine Coalition: The case for personalized medicine (2006), available online at http://www.personalizedmedicinecoalition.org/ communications/pmc_pub_11_06.php

55.28 E. Fix, J.L. Hodges: Discriminatory analysis: Nonparametric discrimination: Consistency properties (UASF School of Aviation Medicine, Randolph Field, 1951)

Please supply the publisher's location.

Please provide the volume number
55.29 V. Vapnik: Statistical Learning Theory (WileyInterscience, New York 1998)

55.30 N. Kasabov: To spike or not to spike: A probabilis tic spiking neuron model, Neural Netw. 23, 16-19 (2010)

55.31 S. Barber. Al: Neural Network for beginners (Part 1 of 3) (2007), available online at http://www. codeproject.com/KB/recipes/NeuralNetwork_1.aspx

55.32 W. Maass: Networks of spiking neurons: The third generation of neural network models, Neural Netw. 10, 1659-1671 (1997)

55.33 A.L. Hodgkin, A.F. Huxley: A quantitative description of membrane current and its application to conduction and excitation in nerve, J. Physiol. 117, 500-544 (1952)

55.34 W. Gerstner: Time structure of the activity of neural network models, Phys. Rev. 51, 738-758 (1995)

55.35 W. Gerstner, W.M. Kistler: Neuron Models: Single Neurons, Populations, Plasticity (Cambridge Univ. Press, Cambridge 2002)

55.36 W. Kistler, W. Gerstner: Reduction of HodgkinHuxley equations to a single variable threshold model, Neural Comput. 9, 1015-1045 (1997)

55.37 W. Maass, C.M. Bishop: Pulsed Neural Networks (MIT Press, Cambridge 1999)

55.38 E.M. Izhikevich: Which model to use for cortical spiking neurons, IEEE Trans. Neural Netw. 15, 10631070 (2004)

55.39 E.M. Izhikevich: Dynamical Systems in Neuroscience (MIT Press, Cambridge 2007)

55.40 E.M. Izhikevich, G.M. Edelman: Large-scale model of mammalian thalamocortical systems, Proc. Natl. Acad. Sci. USA Ts ${ }^{\mathbf{8}}$ 3593-3598 (2008)

55.41 W.C. Yau, et al. Ts ${ }^{6}$ : Visual recognition of speech consonants using facial movement features, Integr. Comput.-Aided Eng. 14, 49-61 (2007)

55.42 C. Fyfe, et al. Ts ${ }^{6}$ : Topological mappings of video and audio data, Int. J. Neural Syst. 18, 481-489 (2008)

55.43 N. Tsapatsoulis, et al. $\mathrm{TS}^{6}$ : An embedded saliency map estimator scheme: Application to video encoding, Int. J. Neural Syst. 17, 289-304 (2007)

55.44 N.C. Schneider, D. Graupe: A modified lamstar neural network and its applications, Int. J. Neural Syst. 18, 331-337 (2008)

55.45 V. Gant, et al. Ts $^{6}$ : Artificial Neural Networks: Practical Considerations for Clinical Applications. In: Clinical Applications of Neural Networks, ed. by R. Dybowski, V. Gant (Cambridge Univ. Press, Cambridge 2001)

55.46 T. Kiyan, T. Yildirim: Breast cancer diagnosis using statistical neural networks, Turk. Symp. Artif. Intell. Neural Netw. (Istanbul 2003)

55.47 P.J. Cowburn, et al. Ts $^{6}$ : Risk stratification in chronic heart failure, Eur. Heart J. 19, 696-710 (1996)

55.48 E. Bartfay, et al. TS $^{6}$ : Comparing the predictive value of neural network models to logistic regres-

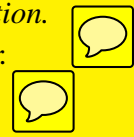


sion models on the risk of death for small-cell lung cancer patients, Eur. J. Cancer Care 15, 115-124 (2006)

55.49 T. Anita, et al. TS ${ }^{6}$ : Early diagnosis of ischemia stroke using neural network, 2009 Proc. Int. Conf. Man-Machine Syst. (ICoMMS) (Batu Ferringhi, Penang 2009)
55.50 K. Kaczmarczyk et al. Ts ${ }^{6}$ : Gait classification in post-stroke patients using artificial neural networks, Gait \& Posture 30, 207-210 (2009)

55.51 S.G. Wysoski, et al. TS $^{6}$ : On-line learning with structural adaptation in a network of spiking neurons for visual pattern recognition, 2006 Int. Conf. Artif. Neural Netw., Lecture Notes in Computer Science (Heidelberg, Berlin 2006) pp. 61-70

55.52 U.M. Fayyad, G.G. Grinstein: Information Visualization in Data Mining and Knowledge Discovery (Morgan Kaufmann, Los altos 2001) 


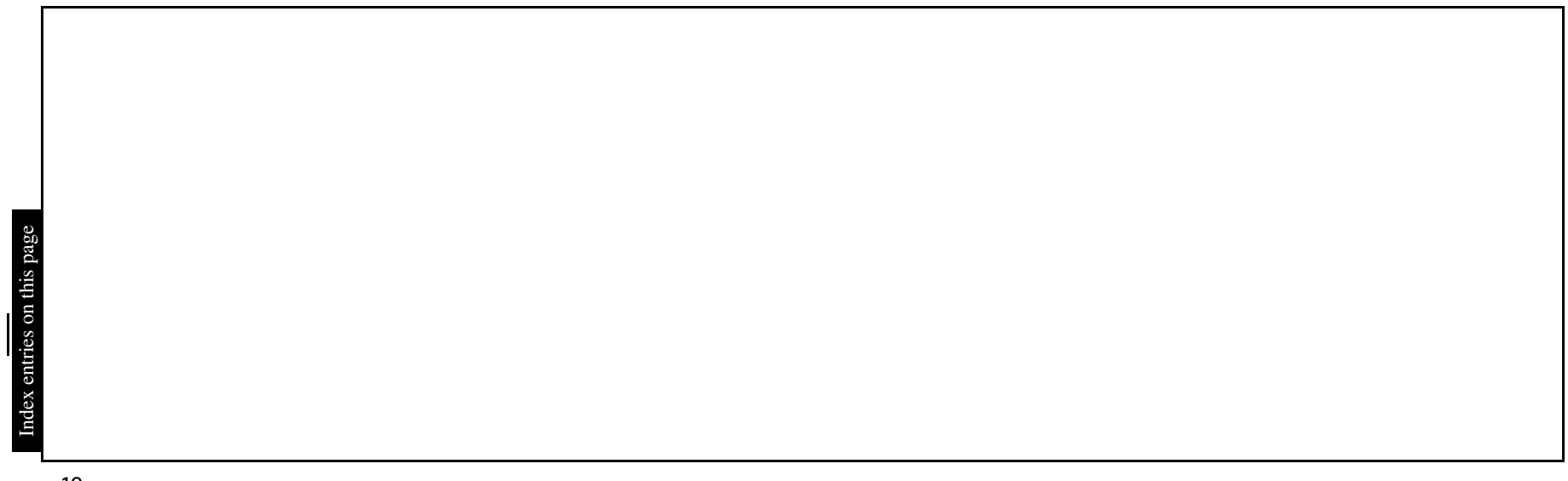

